\title{
Comparison of Surrogate Parameters between CF-Patients in Frankfurt and Moscow (1990-2015)
}

\author{
Jean-Pascal Varescon'1, Christina Smaczny1, Olaf Eickmeier', Gulja Babadjanova², Yulia \\ Philippova $^{2}$, Stanislav Krasovskiy ${ }^{2}$, Elena Amelina ${ }^{2}$ and Thomas Otto Friedrich Wagner ${ }^{1}$ \\ ${ }^{1}$ Christiane Herzog CF-Zentrum, Pneumologie - Klinikum der Goethe-Universität, Germany \\ ${ }^{2}$ Pulmonology Scientific Research Institute - Lomonosov State University Moscow, Russia
}

*Corresponding author: Jean-Pascal Varescon, Christiane Herzog CF-Zentrum, Pneumologie - Klinikum der GoetheUniversität, Theodor-Stern-Kai 7, Haus 18-EG, 60596 Frankfurt am Main, Germany, Tel: +4969-6301-4232

\begin{abstract}
Background: Previous studies have demonstrated that CF prognosis is dependent of three major parameters: FEV1, $\mathrm{BMI}$ and need of intravenous antibiotic therapy. The CF centres of Frankfurt, Germany, and Moscow, Russia, care for cystic fibrosis patients. We decided to investigate and compare both centers from 1990 to 2015. No comparable study has been published so far.
\end{abstract}

Methods: German patient data was collected from the national cystic fibrosis database "Muko.web". Missing values were extracted from the Hospital Information System. Russian patient data were taken directly from the medical records in Moscow. In a descriptive statistical analysis with Bias and RStudio the values were compared.

Results: 428 patients from Moscow (217 male, 211 female; $348(81.3 \%)$ were $P$. aeruginosa positive) and 159 patients from Frankfurt (92 male, 67 female; 137 (86.2\%) with $P$. aeruginosa positive) were compared with regard to $P$. aeruginosa positivity, BMI, FEV1 and need of intravenous antibiotic therapy. CF patients in Moscow stratified by age groups had lower BMI than CF patients in Frankfurt (age 1618: $p=0.003$; age 19-22: $p=0.004 ;$ age 23-29: $p<0.001$; age 30-35: $p<0.001$; age 36-66: $p=0.024)$. In a matching pairs analysis including 100 patients from Frankfurt and 100 patients from Moscow for the year 2015 FEV1 was significantly lower in Moscow patients $(p<0.001)$.

Conclusions: This study showed a significant difference in prognostic parameters between Frankfurt and Moscow in the cross-sectional analysis for the year 2015. A further study should evaluate this difference to show whether this difference will be found over a longer period of time.

\section{Keywords}

Cystic fibrosis, BMI, FEV1, Intravenous antibiotic therapy, Lung function, $P$. aeruginosa, Surrogate parameters

\section{Introduction}

Cystic fibrosis (CF) is a disease characterized by a loss of function of the cystic fibrosis transmembrane conductance regulator (CFTR) in different organs [1]. Previous work has showed that prognosis in $\mathrm{CF}$ is related to Body-Mass-Index (BMI), Forced Expiratory Volume in 1 second (FEV1) and need of intravenous antibiotic therapy [2], this is why these three parameters should be measured and monitored regularly. They have significant impact on survival and on quality of life of CF patients [3]. Disease progression in cystic fibrosis (CF) is marked by deterioration of a number of physiological systems [3] especially lung function is affected [2] progressively leading to pulmonary damage and in a final state to respiratory failure.

The two centers of the Pulmonology Scientific Research Institute, Moscow and the University Hospital Frankfurt (Christiane Herzog CF-Zentrum) started a collaboration starting in July 2018. Both centers care for adult CF-patients, children as well as adults.

A retrospective descriptive study was started to look for differences between patients treated in the Moscow CF center and the Frankfurt CF center from 1990 to 2015. If there was a significant and relevant difference this should be detected in a difference in the three surrogate markers of prognosis in CF: BMI [4], FEV1 [5] and the necessity of intravenous antibiotic therapy caused by exacerbations in CF [6]. BMI can be compared in stratified age classes. Higher BMI is related to better lung function test results (which improves consequently

Citation: Varescon JP, Smaczny C, Eickmeier O, Babadjanova G, Philippova Y, et al. (2021) Comparison of Surrogate Parameters between CF-Patients in Frankfurt and Moscow (1990-2015). Int J Rare Dis Disord 4:031. doi.org/10.23937/2643-4571/1710031

Accepted: July 22, 2021; Published: July 24, 2021

Copyright: (c) 2021 Varescon JP, et al. This is an open-access article distributed under the terms of the Creative Commons Attribution License, which permits unrestricted use, distribution, and reproduction in any medium, provided the original author and source are credited. 
quality of life and survival) and in particular for underweight individuals a poorer prognosis has been reported [4]. FEV1 is the second parameter used to mark progression of CF lung disease progression and evaluate therapeutic efficacy [5]. Furthermore FEV1 is used as prognostic tool for mortality [5,7-9]. A third marker for the survival of CF patients is the need of intravenous antibiotic therapy as a result of a severe pulmonal exacerbation [6] or $P$. aeruginosa infection [10]. Exacerbations have a big consequence in terms of current morbidity as well as implications for long term morbidity and mortality $[6,8]$. P. aeruginosa presence is associated with faster rates of lung function decline in all age groups [10]. Need of intravenous antibiotic therapy consequently results in decreased survival.

No comparable study has been published so far, i.e. there has been no published comparative descriptive study comparing CF patients in different settings in the past.

\section{Materials and Methods}

\section{German patient data}

German patient data were collected from the German national CF registry "muko.web" [11]. This registry was started 1995 under the name "Qualitätssicherung Mukoviszidose" has been renamed "muko.web". In the year 2015 ninety German CF centers took part in data gathering within Muko.web describing in much detail 5331 patients (median age 20; 56.5\% adults; $51.8 \%$ men; 80 died in 2015; median dying age 32) [11]. Data collected from muko.web for the study were height, weight, BMI, FEV1, Forced Vital Capacity (FVC), year of birth, year of death, gender and $P$. aeruginosa presence. In addition to this date of diagnosis of $P$. aeruginosa infection of CF-patients in Frankfurt from 1990 to 2015 were retrieved. These data were anonymized and gathered into an Excel table. Missing values - in particular those describing the utilization of intravenous antibiotic therapy (not listed in muko.web) - were completed with data from the Hospital Medical record Information System (Orbis, Agfa) of the Frankfurt University Hospital. German patients were coded with the letter " $f$ " and were associated to group 01. They received a three-digit numerical code "XXX".

\section{Russian patient data}

Russian patient data were collected directly from the medical files of the Pulmonology Scientific Research Institute, Moscow of the FMBA (Federal MedicalBiological Agency) of Russian Federation. Collected data were anonymized and regrouped in the same standardized table as in Frankfurt. Russian patients were coded with the letter " $\mathrm{m}$ " and were associated to group 02. In the same way as in Frankfurt they received a three-digit numerical code "XXX".

\section{Grouping of data}

With this anonymized code data of both centers were aggregated in one data table. Gender information was coded with 01 for male patients and 02 for female patients. Body weight was expressed in kg (kilograms), body height in $\mathrm{cm}$ (centimeters), FEV1 in $\mathrm{mL}$ (milliliters), FVC in $\mathrm{mL}$ (milliliters). The presence of $P$. aeruginosa was coded with 01 , the absence of $P$. aeruginosa with 02 . Necessity of intravenous antibiotic treatment received the code 01 (02 coded not necessary intravenous antibiotic treatment).

\section{Data analysis with Bias and RStudio}

The complete data were biometrically analyzed with the program "Bias" [12]. After a descriptive approach of complete data, differences in BMI and FEV1 values were observed. Exemplarily BMI in the two centers was compared for 2015 after separating the sample in age classes $[13,14]$. FEV1 is an inconstant value, as it depends on age, height and sex category $[15,16]$. To compare FEV1 between both centers in 2015 a program was written in Rcode and executed with RStudio - a statistical programming tool, which can execute Rcode and analyze statistical data. Matchlt [17] was used to create two new comparable samples. They were matched according to the parameters influencing FEV1 (height, age, sex category) $[15,16]$. The size of both samples was 100 patients and both samples were statistically not significant different (before matching $p$ $<0.001$, after matching $p=0.484)$. Afterward both new samples were compared for their FEV1 values in a new statistical biometrical analysis with "Bias" [12].

\section{Comparison to normal population}

At the end the descriptive data of the study were compared to normal population data in Germany [18] and the Russian Federation [19-21] taking in consideration epidemiological differences, which can influence CFpatients in both centers. Especially differences in BMI in both countries may have an influence on samples BMI.

\section{Results}

\section{Description of the data range: Year of birth}

The study totalized 428 (72.91\%) patients from Moscow and 159 (27.09\%) patients from Frankfurt, what conducted to a total of 587 analyzed patients. All of them were born in 1999 or earlier. The oldest patient of this study was born in 1949 (Figure 1).

Average [22] year of birth was 1982 for Frankfurt, 1988 for Moscow and for the total cohort 1986. Standard deviation [22] was 11.10 for Frankfurt, 6.42 for Moscow and 8.45 for total cohort. Median [22] year of birth was 1983 for Frankfurt ( $1^{\text {st }}$ quartile $1973,3^{\text {rd }}$ quartile 1992 ), 1989 for Moscow ( $1^{\text {st }}$ quartile $1985,3^{\text {rd }}$ quartile 1993) and 1988 for total cohort ( $1^{\text {st }}$ quartile $1983,3^{\text {rd }}$ quartile 1993). Minimum in Frankfurt was 1956. In Moscow it 

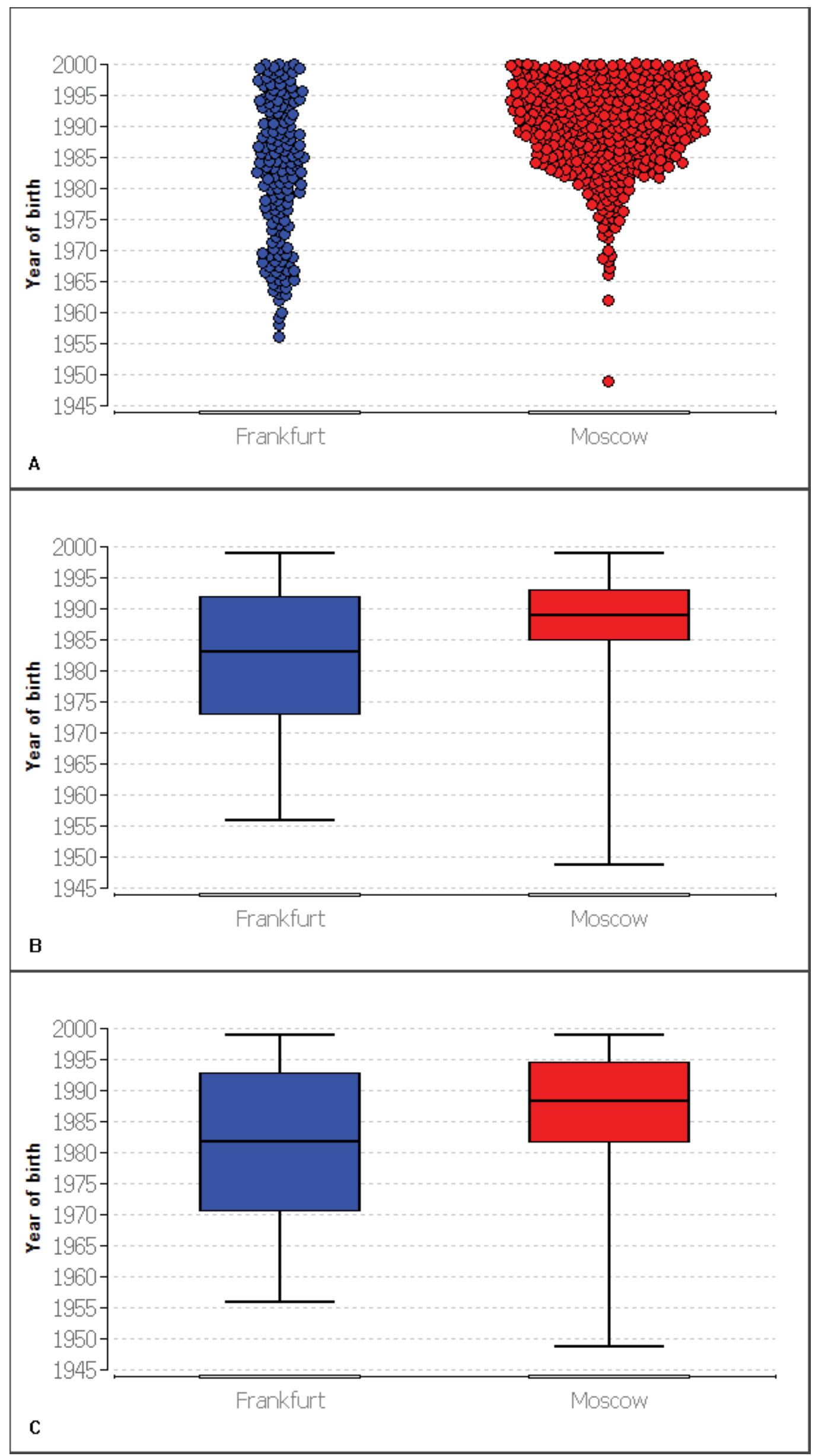

Figure 1: Differences in year of birth in both CF-populations (A) Year of birth distribution of CF-patients in both centers described in dot plots; (B) Box plot of patients year of birth representing median, first quartile and third quartile; (C) Box plot of patients year of birth representing average and standard deviation. 
was 1949 (consequently 1949 for total cohort). In both centers maximum was 1999 (so same for total cohort. Finally range was 43 for Frankfurt and 50 for Moscow and total cohort.

\section{Description of the data range: Gender distribution, $P$. aeruginosa presence and more}

In Moscow 217 (50.70\%) male patients and 211 (49.30\%) female patients were counted, while in Frankfurt 92 (57.86\%) male patients and 67 (42.14\%) female patients were registered. A performed Chisquare test with Yates's correction for continuity [22] confirms both samples were comparable $(p=0.147)$ for sex category distribution.

348 (81.31\%) Moscow patients were infected with $P$. aeruginosa and $80(18.69 \%)$ were not infected with this bacterium. In Frankfurt the number of $P$. aeruginosa infected patients was 137 (86.16\%) while 22 CF patients were not infected (13.84\%). In the same way as for the sex category distribution a Chi-square test with Yates's correction for continuity [22] was performed ( $p=$ 0.209). Subsequently both CF-patient populations were comparable for $P$. aeruginosa infections $(p=0.209)$.

$6(3.77 \%)$ recorded patients died in Frankfurt (0 until 2015) and 114 (26.64\%) recorded patients died in Moscow (68 (15.89\%) until 2015).

\section{Description of the data range: BMI, FEV1 and necessity of intravenous antibiotic therapy evolution over time}

Data were statistically analyzed and important values were calculated and entered into Table 1. Key values of BMI, FEV1 and necessity of intravenous antibiotic therapy were examined.

Table 1: A) BMI biometrical descriptive statistic from 1990 to 2015 including number of patient data sets, average BMI, median $\mathrm{BMI}, \mathrm{SD}$ (standard deviation) BMI, maximum BMI, minimum BMI, BMI range, $1^{\text {st }}$ quartile BMI and $3^{\text {rd }}$ quartile BMI.

\begin{tabular}{|c|c|c|c|c|c|c|}
\hline \multirow[t]{2}{*}{ Year } & \multicolumn{2}{|c|}{ Number of Patient data } & \multicolumn{2}{|c|}{ BMI average } & \multicolumn{2}{|c|}{ BMI median } \\
\hline & Frankfurt & Moscow & Frankfurt & Moscow & Frankfurt & Moscow \\
\hline 1990 & 2 & 0 & 21.52 & - & 21.52 & - \\
\hline 1991 & 2 & 1 & 21.54 & 14.49 & 21.54 & 14.49 \\
\hline 1992 & 1 & 2 & 21.50 & 16.27 & 21.50 & 16.27 \\
\hline 1993 & 2 & 7 & 20.91 & 16.44 & 20.91 & 15.08 \\
\hline 1994 & 1 & 11 & 20.02 & 17.34 & 20.02 & 17.16 \\
\hline 1995 & 10 & 7 & 16.66 & 17.36 & 16.11 & 17.16 \\
\hline 1996 & 30 & 10 & 18.84 & 16.91 & 18.52 & 17.39 \\
\hline 1997 & 35 & 23 & 19.41 & 17.61 & 19.55 & 17.72 \\
\hline 1998 & 46 & 38 & 19.89 & 16.34 & 19.66 & 16.45 \\
\hline 1999 & 45 & 39 & 19.97 & 17.33 & 19.13 & 17.65 \\
\hline 2000 & 30 & 45 & 20.29 & 16.98 & 19.09 & 16.53 \\
\hline 2001 & 14 & 53 & 19.07 & 16.79 & 18.05 & 16.85 \\
\hline 2002 & 16 & 64 & 18.78 & 17.41 & 18.02 & 17.54 \\
\hline 2003 & 68 & 78 & 21.43 & 17.42 & 20.85 & 17.55 \\
\hline 2004 & 75 & 103 & 21.48 & 17.49 & 20.76 & 17.57 \\
\hline 2005 & 13 & 101 & 20.57 & 18.04 & 20.68 & 18.03 \\
\hline 2006 & 13 & 124 & 20.95 & 18.02 & 21.27 & 17.96 \\
\hline 2007 & 13 & 160 & 20.51 & 18.11 & 21.10 & 18.13 \\
\hline 2008 & 91 & 179 & 21.69 & 18.38 & 21.01 & 18.55 \\
\hline 2009 & 84 & 188 & 22.39 & 18.55 & 21.81 & 18.52 \\
\hline 2010 & 132 & 192 & 21.20 & 18.76 & 20.70 & 18.69 \\
\hline 2011 & 137 & 199 & 21.40 & 18.71 & 21.14 & 18.47 \\
\hline 2012 & 131 & 250 & 21.80 & 18.79 & 21.62 & 18.51 \\
\hline 2013 & 130 & 263 & 21.99 & 18.68 & 21.66 & 18.29 \\
\hline 2014 & 133 & 278 & 22.12 & 18.78 & 21.73 & 18.52 \\
\hline 2015 & 141 & 301 & 22.24 & 18.74 & 21.63 & 18.59 \\
\hline \multirow[t]{2}{*}{ Year } & \multicolumn{2}{|c|}{ BMI standard deviation (SD) } & \multicolumn{2}{|c|}{ BMI maximum } & \multicolumn{2}{|c|}{ BMI minimum } \\
\hline & Frankfurt & Moscow & Frankfurt & Moscow & Frankfurt & Moscow \\
\hline 1990 & 0.64 & - & 21.98 & - & 21.07 & - \\
\hline 1991 & 1.13 & - & 22.34 & 14.49 & 20.75 & 14.49 \\
\hline
\end{tabular}




\begin{tabular}{|c|c|c|c|c|c|c|}
\hline 1992 & - & 1.75 & 21.50 & 17.51 & 21.50 & 15.03 \\
\hline 1993 & 1.26 & 2.88 & 21.80 & 20.93 & 20.02 & 13.22 \\
\hline 1994 & - & 3.82 & 20.02 & 25.00 & 20.02 & 13.34 \\
\hline 1995 & 2.29 & 2.37 & 20.64 & 22.21 & 13.68 & 15.43 \\
\hline 1996 & 3.17 & 2.21 & 25.83 & 19.37 & 14.07 & 13.47 \\
\hline 1997 & 3.18 & 2.49 & 28.22 & 22.77 & 13.71 & 13.34 \\
\hline 1998 & 3.18 & 3.58 & 27.64 & 22.77 & 13.65 & 1.92 \\
\hline 1999 & 3.52 & 2.51 & 31.11 & 22.94 & 14.88 & 12.63 \\
\hline 2000 & 4.89 & 2.71 & 37.56 & 24.15 & 13.13 & 12.70 \\
\hline 2001 & 4.02 & 2.69 & 27.76 & 22.76 & 14.60 & 12.40 \\
\hline 2002 & 2.94 & 2.65 & 24.01 & 23.23 & 14.74 & 11.65 \\
\hline 2003 & 3.76 & 2.70 & 33.30 & 23.61 & 13.98 & 11.65 \\
\hline 2004 & 4.05 & 2.77 & 35.50 & 24.88 & 12.93 & 10.82 \\
\hline 2005 & 3.22 & 2.76 & 26.35 & 25.86 & 13.73 & 12.02 \\
\hline 2006 & 3.35 & 2.83 & 26.67 & 25.72 & 14.38 & 12.03 \\
\hline 2007 & 3.58 & 2.73 & 24.97 & 25.62 & 13.89 & 12.73 \\
\hline 2008 & 4.10 & 2.79 & 40.75 & 26.23 & 14.38 & 11.83 \\
\hline 2009 & 4.41 & 2.78 & 44.29 & 26.03 & 15.34 & 12.80 \\
\hline 2010 & 4.20 & 2.84 & 45.35 & 30.03 & 14.27 & 12.60 \\
\hline 2011 & 4.18 & 2.90 & 45.52 & 31.99 & 14.35 & 12.47 \\
\hline 2012 & 4.03 & 2.69 & 45.34 & 27.73 & 13.86 & 12.47 \\
\hline 2013 & 4.04 & 2.80 & 44.47 & 27.73 & 13.86 & 10.85 \\
\hline 2014 & 4.17 & 2.86 & 45.41 & 31.46 & 14.10 & 13.02 \\
\hline 2015 & 4.13 & 2.78 & 46.60 & 31.46 & 14.17 & 11.33 \\
\hline Year & \multicolumn{2}{|c|}{ BMI range } & \multicolumn{2}{|c|}{ BMI $1^{\text {st }}$ quartile } & \multicolumn{2}{|c|}{ BMI $3^{\text {rd }}$ quartile } \\
\hline & Frankfurt & Moscow & Frankfurt & Moscow & Frankfurt & Moscow \\
\hline 1990 & 0.91 & - & - & - & - & - \\
\hline 1991 & 1.59 & 0.00 & - & - & - & - \\
\hline 1992 & 0.00 & 2.48 & - & - & - & - \\
\hline 1993 & 1.78 & 7.71 & - & 14.49 & - & 18.42 \\
\hline 1994 & 0.00 & 11.66 & - & 14.22 & - & 19.30 \\
\hline 1995 & 6.96 & 6.78 & 15.21 & 15.64 & 17.29 & 17.70 \\
\hline 1996 & 11.76 & 5.90 & 16.51 & 15.35 & 20.88 & 18.85 \\
\hline 1997 & 14.51 & 9.43 & 17.38 & 15.89 & 20.87 & 19.12 \\
\hline 1998 & 13.99 & 20.85 & 17.93 & 14.22 & 21.60 & 18.46 \\
\hline 1999 & 16.23 & 10.32 & 17.79 & 15.41 & 21.72 & 19.00 \\
\hline 2000 & 24.43 & 11.45 & 17.59 & 14.81 & 21.15 & 19.23 \\
\hline 2001 & 13.16 & 10.36 & 15.90 & 14.66 & 21.14 & 18.67 \\
\hline 2002 & 9.27 & 11.58 & 16.76 & 15.23 & 21.38 & 18.93 \\
\hline 2003 & 19.33 & 11.96 & 19.03 & 15.23 & 22.92 & 19.11 \\
\hline 2004 & 22.57 & 14.06 & 19.23 & 15.21 & 23.00 & 19.47 \\
\hline 2005 & 12.61 & 13.85 & 19.33 & 16.37 & 22.01 & 19.68 \\
\hline 2006 & 12.29 & 13.69 & 19.76 & 15.66 & 22.60 & 19.82 \\
\hline 2007 & 11.08 & 12.89 & 20.48 & 16.28 & 22.92 & 19.91 \\
\hline 2008 & 26.37 & 14.40 & 19.58 & 16.47 & 22.80 & 20.09 \\
\hline 2009 & 28.95 & 13.22 & 20.03 & 16.71 & 23.46 & 19.93 \\
\hline 2010 & 31.08 & 17.43 & 18.81 & 16.97 & 22.95 & 20.20 \\
\hline 2011 & 31.17 & 19.53 & 19.05 & 16.93 & 23.13 & 20.45 \\
\hline 2012 & 31.48 & 15.27 & 19.34 & 16.86 & 23.29 & 20.45 \\
\hline 2013 & 30.61 & 16.88 & 19.58 & 16.82 & 23.69 & 20.43 \\
\hline 2014 & 31.32 & 18.44 & 19.31 & 16.86 & 23.81 & 20.50 \\
\hline 2015 & 32.43 & 20.13 & 19.31 & 16.82 & 24.14 & 20.32 \\
\hline
\end{tabular}


B) FEV1 biometrical descriptive statistic from 1990 to 2015 including number of patient data, average FEV1, median FEV1, SD (standard deviation) FEV1, maximum FEV1, minimum FEV1, FEV1 range, $1^{\text {st }}$ quartile FEV1 and $3^{\text {rd }}$ quartile FEV1.

\begin{tabular}{|c|c|c|c|c|c|c|}
\hline \multirow[t]{2}{*}{ Year } & \multicolumn{2}{|c|}{ Number of Patient data } & \multicolumn{2}{|c|}{ FEV1 average } & \multicolumn{2}{|c|}{ FEV1 median } \\
\hline & Frankfurt & Moscow & Frankfurt & Moscow & Frankfurt & Moscow \\
\hline 1990 & 2 & 0 & 3800 & - & 3800 & - \\
\hline 1991 & 2 & 0 & 3590 & - & 3590 & - \\
\hline 1992 & 1 & 1 & 4370 & 2820 & 4370 & 2820 \\
\hline 1993 & 2 & 4 & 3600 & 1280 & 3600 & 1000 \\
\hline 1994 & 1 & 8 & 2550 & 1706.25 & 2550 & 1210 \\
\hline 1995 & 6 & 6 & 2136.67 & 1216.67 & 2030 & 1155 \\
\hline 1996 & 27 & 6 & 1825.93 & 1783.33 & 1800 & 1685 \\
\hline 1997 & 34 & 19 & 2150.88 & 1998.42 & 2070 & 1640 \\
\hline 1998 & 42 & 24 & 2357.62 & 1783.33 & 2090 & 1350 \\
\hline 1999 & 44 & 28 & 2244.32 & 2215.00 & 2205 & 1980 \\
\hline 2000 & 28 & 25 & 2512.86 & 2193.20 & 2555 & 2300 \\
\hline 2001 & 13 & 30 & 2333.85 & 2019.67 & 2120 & 1955 \\
\hline 2002 & 17 & 37 & 2328.82 & 2026.76 & 2090 & 1920 \\
\hline 2003 & 66 & 50 & 2439.39 & 2186.00 & 2320 & 2000 \\
\hline 2004 & 71 & 67 & 2447.89 & 2221.34 & 2340 & 2020 \\
\hline 2005 & 15 & 73 & 2366.67 & 2296.71 & 2350 & 2030 \\
\hline 2006 & 13 & 87 & 2571.54 & 2202.41 & 2450 & 2040 \\
\hline 2007 & 13 & 125 & 2728.46 & 2301.36 & 2480 & 2130 \\
\hline 2008 & 93 & 155 & 2354.73 & 2247.81 & 2230 & 2010 \\
\hline 2009 & 86 & 171 & 2488.72 & 2299.30 & 2450 & 2220 \\
\hline 2010 & 133 & 169 & 2463.91 & 2270.77 & 2270 & 2120 \\
\hline 2011 & 137 & 176 & 2446.93 & 2192.33 & 2300 & 2090 \\
\hline 2012 & 133 & 234 & 2437.44 & 2136.54 & 2230 & 2030 \\
\hline 2013 & 135 & 251 & 2450.67 & 2120.84 & 2370 & 2020 \\
\hline 2014 & 135 & 275 & 2426.81 & 2057.35 & 2310 & 1900 \\
\hline 2015 & 145 & 295 & 2460.34 & 1983.12 & 2290 & 1850 \\
\hline \multirow[t]{2}{*}{ Year } & \multicolumn{2}{|c|}{ FEV1 standard deviation (SD) } & \multicolumn{2}{|c|}{ FEV1 maximum } & \multicolumn{2}{|c|}{ FEV1 minimum } \\
\hline & Frankfurt & Moscow & Frankfurt & Moscow & Frankfurt & Moscow \\
\hline 1990 & 565.69 & - & 4200 & - & 3400 & - \\
\hline 1991 & 1343.50 & - & 4540 & - & 2640 & - \\
\hline 1992 & - & - & 4370 & 2820 & 4370 & 2820 \\
\hline 1993 & 1173.80 & 671.71 & 4430 & 2280 & 2770 & 840 \\
\hline 1994 & - & 1290.10 & 2550 & 4390 & 2550 & 610 \\
\hline 1995 & 638.55 & 647.79 & 3320 & 2420 & 1520 & 600 \\
\hline 1996 & 512.72 & 1070.49 & 3090 & 3180 & 920 & 630 \\
\hline 1997 & 726.81 & 1160.03 & 3960 & 4060 & 870 & 610 \\
\hline 1998 & 883.31 & 1036.85 & 4400 & 3820 & 1090 & 570 \\
\hline 1999 & 821.57 & 1126.85 & 4310 & 5020 & 130 & 580 \\
\hline 2000 & 906.30 & 948.37 & 4080 & 3810 & 900 & 870 \\
\hline 2001 & 1041.78 & 1000.76 & 4330 & 4330 & 560 & 570 \\
\hline 2002 & 1053.90 & 905.15 & 4540 & 4060 & 600 & 660 \\
\hline 2003 & 1027.37 & 1044.13 & 5820 & 4510 & 500 & 600 \\
\hline 2004 & 908.67 & 1063.67 & 4870 & 4790 & 570 & 480 \\
\hline 2005 & 1074.40 & 1141.05 & 4530 & 6490 & 920 & 460 \\
\hline 2006 & 930.45 & 1036.96 & 4530 & 5980 & 960 & 610 \\
\hline 2007 & 1053.31 & 1043.37 & 4590 & 5650 & 1340 & 510 \\
\hline
\end{tabular}




\begin{tabular}{|c|c|c|c|c|c|c|}
\hline 2008 & 971.57 & 1114.60 & 5030 & 5870 & 580 & 380 \\
\hline 2009 & 1055.56 & 1073.32 & 5440 & 6240 & 560 & 480 \\
\hline 2010 & 1036.99 & 981.22 & 5390 & 5390 & 710 & 730 \\
\hline 2011 & 1028.96 & 1036.40 & 5440 & 5080 & 650 & 420 \\
\hline 2012 & 1023.65 & 1036.54 & 5130 & 6420 & 580 & 450 \\
\hline 2013 & 1015.53 & 1034.53 & 5300 & 6400 & 690 & 500 \\
\hline 2014 & 1042.62 & 990.51 & 5370 & 5130 & 730 & 192 \\
\hline 2015 & 1112.38 & 985.01 & 5410 & 5220 & 600 & 520 \\
\hline \multirow[t]{2}{*}{ Year } & \multicolumn{2}{|c|}{ FEV1 range } & \multicolumn{2}{|c|}{ FEV1 $1^{\text {st }}$ quartile } & \multicolumn{2}{|c|}{ FEV1 $3^{\text {rd }}$ quartile } \\
\hline & Frankfurt & Moscow & Frankfurt & Moscow & Frankfurt & Moscow \\
\hline 1990 & 800 & - & - & - & - & - \\
\hline 1991 & 1900 & - & - & - & - & - \\
\hline 1992 & 0 & 0 & - & 2820 & - & 2820 \\
\hline 1993 & 1660 & 1440 & - & 930 & - & 1350 \\
\hline 1994 & 0 & 3780 & - & 870 & - & 1972.5 \\
\hline 1995 & 1800 & 1820 & 1755 & 807.5 & 2200 & 1247.5 \\
\hline 1996 & 2170 & 2550 & 1515 & 892.5 & 2060 & 2590 \\
\hline 1997 & 3090 & 3450 & 1720 & 1090 & 2517.5 & 2975 \\
\hline 1998 & 3310 & 3250 & 1772.5 & 975 & 2897.5 & 2472.5 \\
\hline 1999 & 4180 & 4440 & 1775 & 1357.5 & 2607.5 & 3085 \\
\hline 2000 & 3180 & 2940 & 1760 & 1230 & 3402.5 & 2740 \\
\hline 2001 & 3770 & 3760 & 1520 & 1135 & 3000 & 2655 \\
\hline 2002 & 3940 & 3400 & 1600 & 1280 & 3090 & 2540 \\
\hline 2003 & 5320 & 3910 & 1755 & 1262.5 & 3157.5 & 2807.5 \\
\hline 2004 & 4300 & 4310 & 1805 & 1405 & 2915 & 2865 \\
\hline 2005 & 3610 & 6030 & 1805 & 1540 & 2790 & 2710 \\
\hline 2006 & 3570 & 5370 & 2030 & 1500 & 2870 & 2695 \\
\hline 2007 & 3250 & 5140 & 1920 & 1680 & 3440 & 2840 \\
\hline 2008 & 4450 & 5490 & 1670 & 1370 & 2940 & 2985 \\
\hline 2009 & 4880 & 5760 & 1732.5 & 1455 & 2980 & 3045 \\
\hline 2010 & 4680 & 4660 & 1690 & 1560 & 2980 & 2970 \\
\hline 2011 & 4790 & 4660 & 1680 & 1355 & 2960 & 2890 \\
\hline 2012 & 4550 & 5970 & 1670 & 1332.5 & 3060 & 2820 \\
\hline 2013 & 4610 & 5900 & 1690 & 1315 & 2975 & 2730 \\
\hline 2014 & 4640 & 4938 & 1690 & 1230 & 2895 & 2785 \\
\hline 2015 & 4810 & 4700 & 1600 & 1245 & 3280 & 2585 \\
\hline
\end{tabular}

C) Biometrical descriptive statistical analysis of necessity of intravenous antibiotic therapy from 1990 to 2015 including number of patient data, number of necessity of intravenous antibiotic therapy and percentage of necessity of intravenous antibiotic therapy.

\begin{tabular}{|c|c|c|c|c|}
\hline Year & \multicolumn{2}{|c|}{ Number of Patient data } & \multicolumn{2}{|c|}{ Necessity of intravenous antibiotic therapy } \\
\hline & Frankfurt & Moscow & Frankfurt & Moscow \\
\hline 1990 & 24 & 1 & 1 & 0 \\
1991 & 24 & 2 & 1 & 1 \\
1992 & 25 & 3 & 1 & 2 \\
1993 & 26 & 9 & 1 & 3 \\
1994 & 26 & 15 & 1 & 4 \\
1995 & 27 & 12 & 3 & 4 \\
1996 & 27 & 15 & 2 & 8 \\
1997 & 27 & 26 & 2 & 15 \\
\hline
\end{tabular}




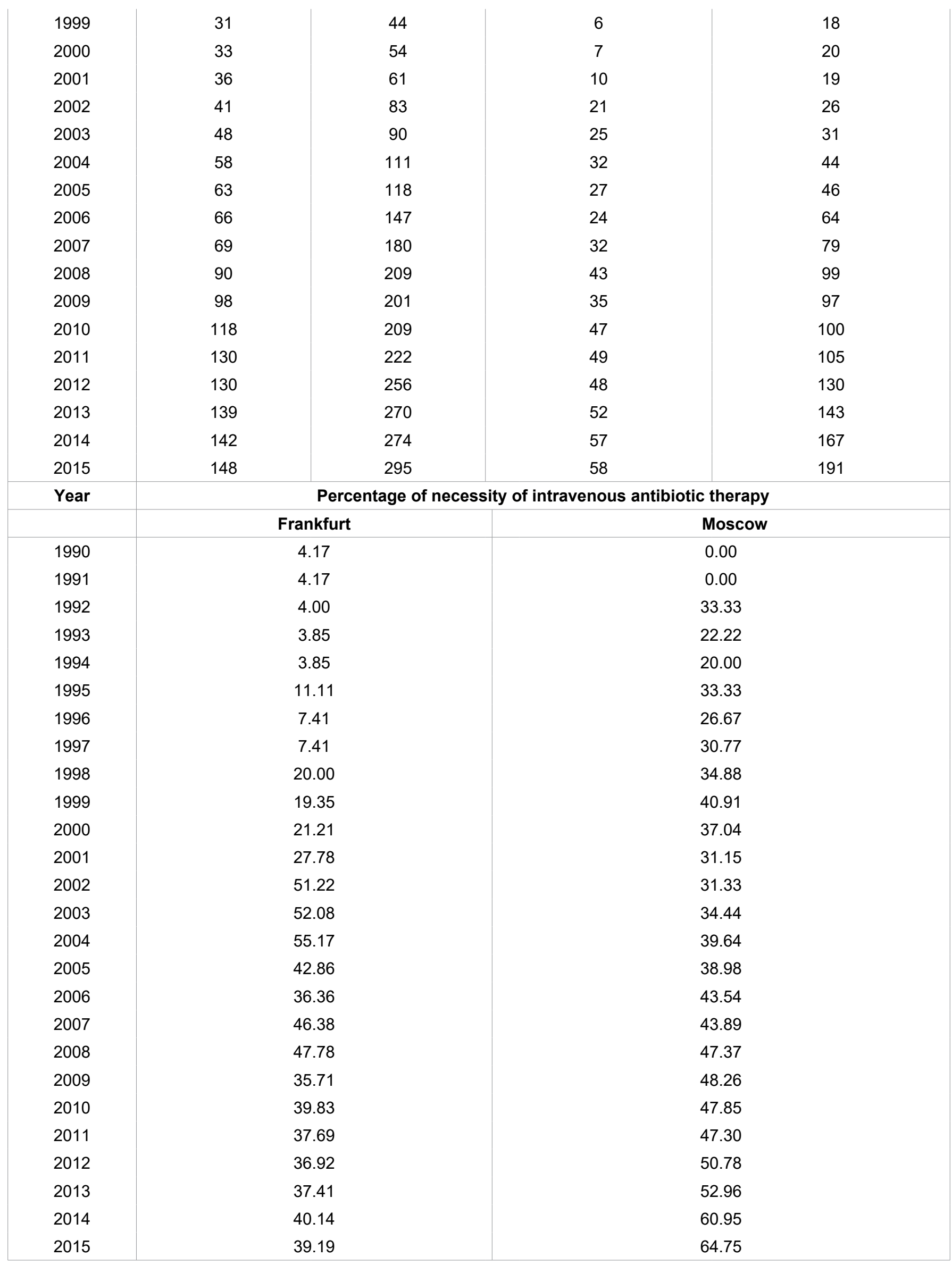




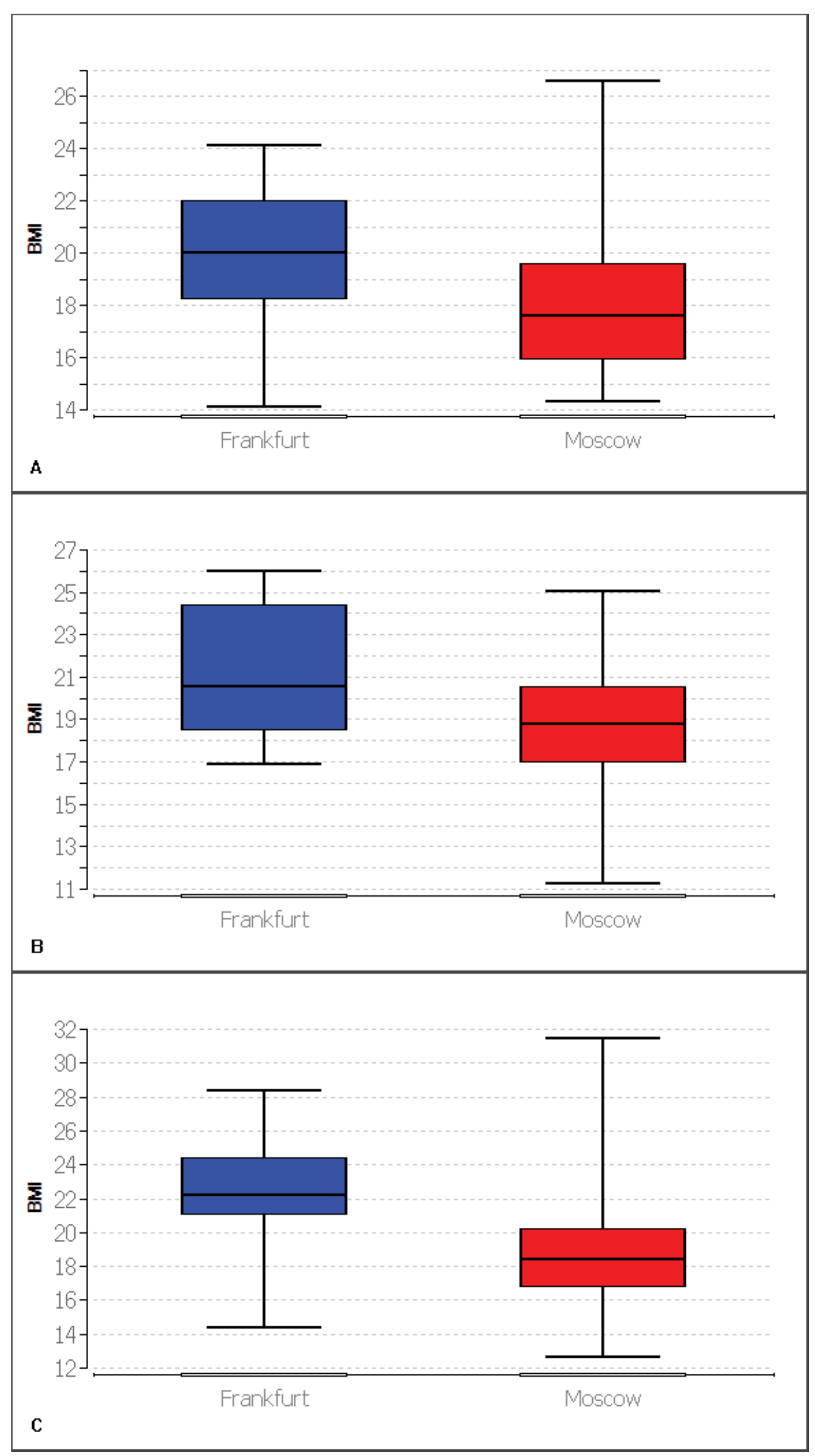

Figure 2: Box plots representing BMI comparison of patients for 2015 with median, first quartile and third quartile (A) Patients aged 16 to 18 years (Average BMl: Frankfurt $(n=12)$ : 19.95; Moscow $(n=25)$ : 17.90); (B) Patients aged 19 to 22 years (Average BMI: Frankfurt $(n=19)$ : 20.87; Moscow $(n=76)$ : 18.75); (C) Patients aged 23 to 29 years (Average BMI: Frankfurt $(n=25)$ : 22.59; Moscow $(n=139): 18.66)$. 
Data were statistically analyzed and values were calculated and entered into Table 1. Key values of BMI, FEV1 and necessity of intravenous antibiotic therapy were examined. In summary the parameters in Table 1 are mostly better for Frankfurt patients than for Moscow patients. From 1990 to 1995 there were not sufficient data and consequently values and results cannot be considered to be representative. FEV1 values have to corrected by height, age and sex category $[15,16]$, therefore, they are not directly comparable.

\section{Statistical BMI comparison of both CF populations in 2015}

To evaluate if there was a significant statistical BMI difference between patients in Frankfurt and Moscow year 2015 was analyzed exemplarily. Patients were categorized in age groups (Figure 2 and Figure 3 ).

In 2015 Moscow CF patients stratified by age groups had statistically significant lower BMI than Frankfurt CF patients in all age groups (age 16-18: $p=0.003$; age 19-
22: $p=0.004$; age 23-29: $p<0.001 ;$ age 30-35: $p<0.001$; age 36-66: $p=0.024)[22,23-25]$.

\section{Statistical FEV1 comparison of matched samples in 2015}

To compare FEV1 in both centers a program run with RStudio [17] allowed isolation of two matched samples by height (before matching $p=0.028$, after matching $p=$ 0.876 ), age (before matching $p<0.001$, after matching $p=$ 0.484 ) and sex category (before matching $p=0.088$, after matching $p=0.258$ ) for 2015. Both included 100 patients (first sample with 100 Frankfurt patients and second sample with 100 Moscow patients) and were comparable after matching. Statistical analysis showed FEV1 was significantly lower for Moscow CF-patients $(p<0.001)$ than for Frankfurt CF-patients in 2015 (Table 2), (Figure 4).

\section{Discussion}

\section{Summary}

Data described both CF-populations in Frankfurt and
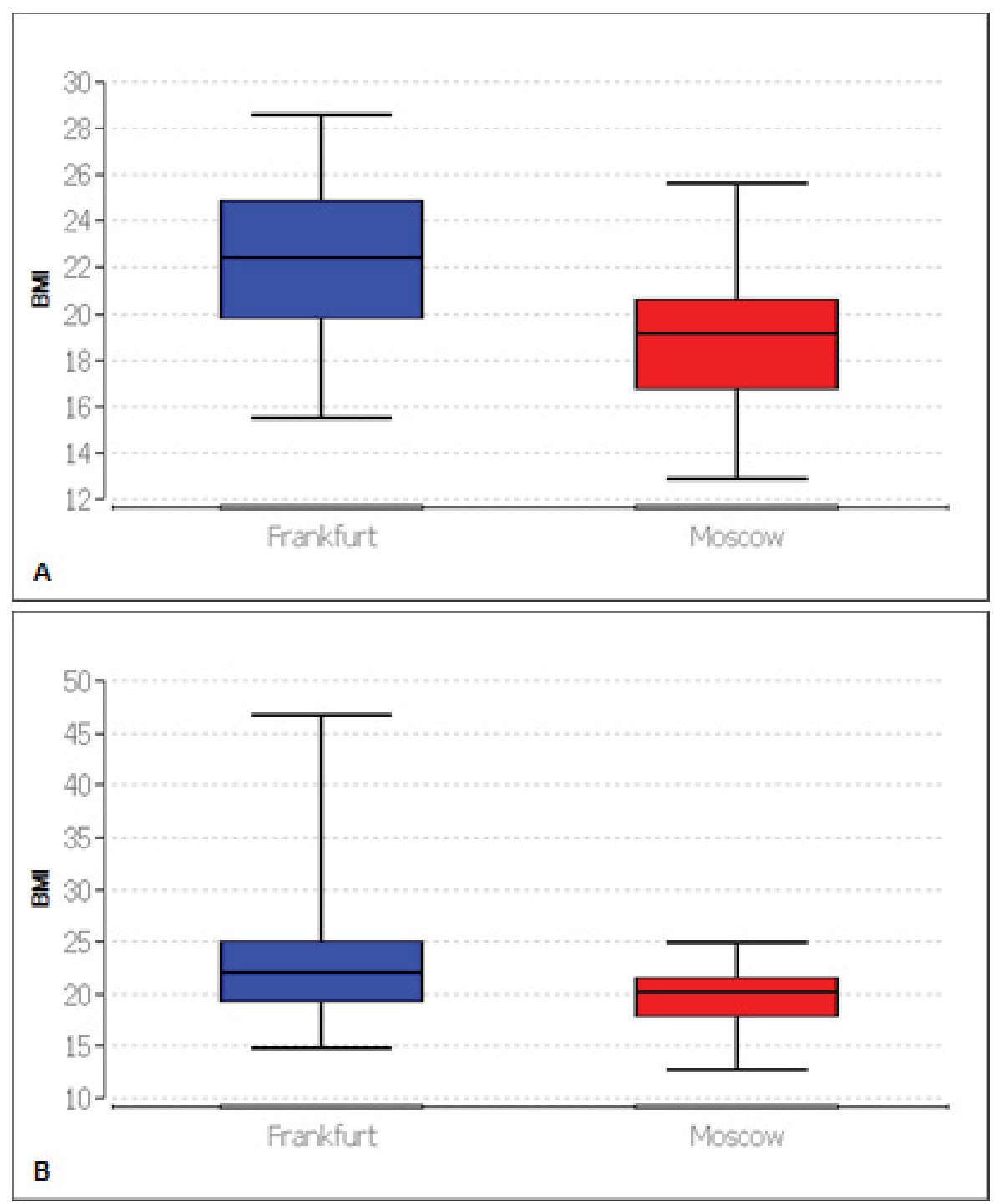

Figure 3: Box plots representing BMI comparison of patients for 2015 with median, first quartile and third quartile (A) Patients aged 30 to 35 years (Average BMI: Frankfurt $(n=29)$ : 22.27; Moscow $(n=44)$ : 18.97); (B) Patients older than 35 years (Average BMI: Frankfurt $(n=56)$ : 23.03; Moscow $(n=17)$ : 19.93). 


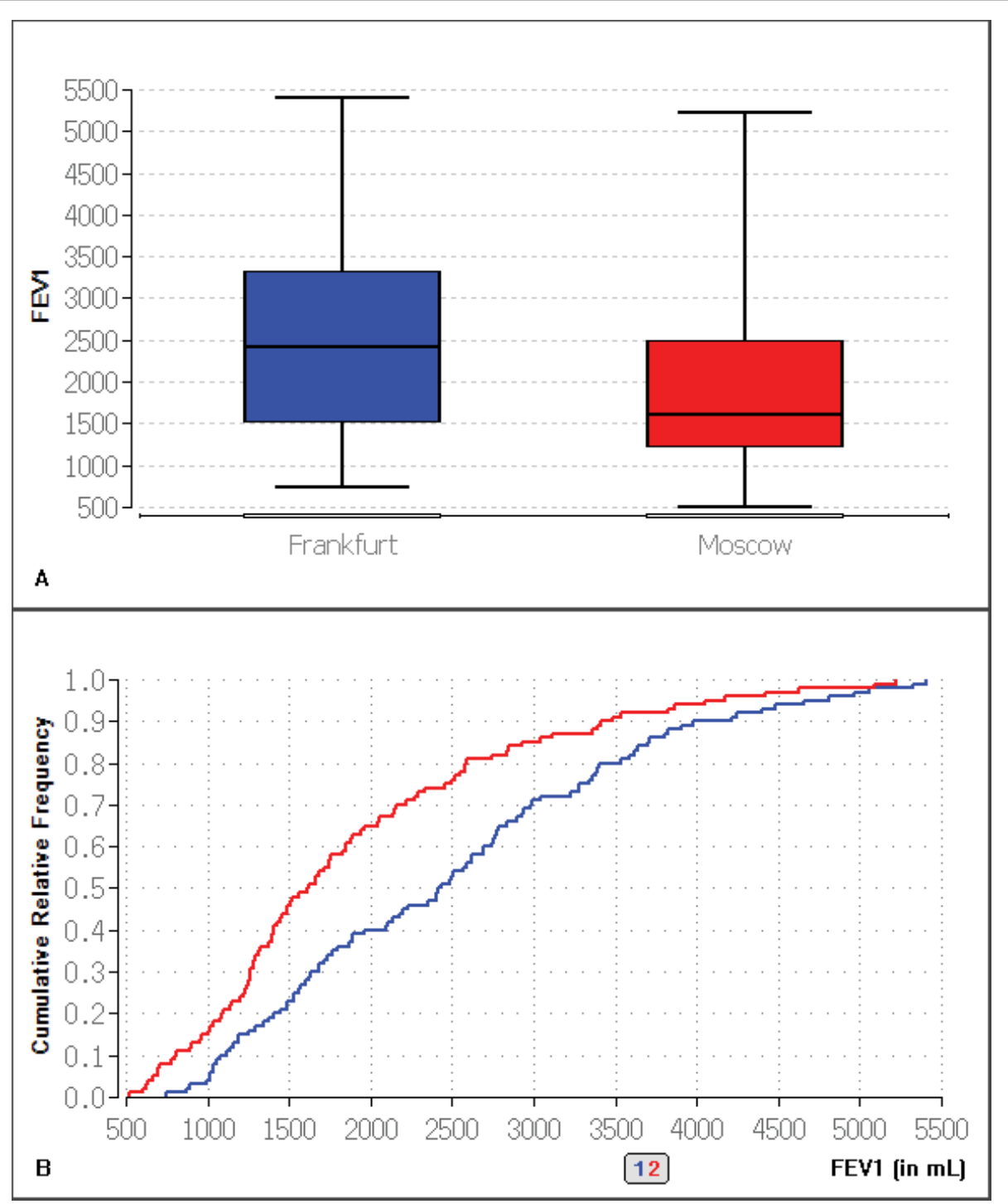

Figure 4: A) Box plot representing FEV1 comparison of both samples for 2015 with median, first quartile and third quartile; B) Empirical distribution function of FEV1 in both samples (blue = Frankfurt, red = Moscow) for 2015.

Table 2: Biometrical statistic analysis of FEV1 in 2015 for both samples ( $n=100$ CF-patients in Frankfurt and $n=100$ CF-patients in Moscow). Average, median, SD, maximum, minimum, range, first quartile and third quartile are higher in Frankfurt than in Moscow.

\begin{tabular}{|l|l|l|l|l|l|l|ll|}
\hline FEV1 & Average & Median & SD & Maximum & Minimum & Range & $\mathbf{1}^{\text {st }}$ quartile & $\mathbf{3}^{\text {rd }}$ quartile \\
\hline Frankfurt & 2497.90 & 2420.00 & 1143.19 & 5410.00 & 750.00 & 4660.00 & 1537.50 & 3325.00 \\
Moscow & 1908.70 & 1615.00 & 1044.80 & 5220.00 & 520.00 & 4700.00 & 1222.50 & 2487.50 \\
\hline
\end{tabular}

Moscow. At first glance values of BMI, FEV1 and the necessity of intravenous antibiotic therapy were better in Frankfurt than in Moscow. An evaluation of both CFpopulations for 2015 revealed BMI was significantly higher in Frankfurt, than in Moscow. A high BMI is a positive predictor for a better outcome $[26,27]$ and decreased mortality [26]. Epidemiological analysis of normal German [18] and Russian [19-21] population didn't explain this severe gap (referred to 3.6.). In the same way both FEV1 populations of 2015 obtained with the R-program to get comparable samples showed Frankfurt CF-patients have a better FEV1 than Moscow CF-patients. A better FEV1 is associated with a better outcome [26] and a lower mortality. Subsequently these data indicate Frankfurt patients should have a better outcome than Moscow patients.

\section{Comparison with BMI and FEV1 of normal population}

At first we had to analyze epidemiological available data of normal population to see if both are reasonably comparable. Latest data from the German federal office of statistics [18] shows a mean BMI of 26.0 for German population in 2017. Russian data [19-21] are not equally detailed and latest data was published in 2014. Mean BMI in the Russian population was 26.5. In the same year mean BMI was 26.3 in Germany, this might mean that the Russian population has a higher mean BMI than the German population, however both populations can be considered comparable. Consequently a possible gap 
in BMI in both CF-populations (referred to 3.4.) cannot be explained by epidemiological data of the normal population.

A comparison of FEV1\% between Germany and the Russian Federation [28] shows a difference for patients categorized in age groups. Average and mean values seem higher in Germany for children and for adults. According to ECFSPR annual report of 2017 [28] the FEV1\% of Germany and the Russian Federation are different. German data seem to resemble the pooled data very closely, while the Russian data seem to be lower than pooled data and German data. This is the reason why we expected differences between both centers we wanted to analyze.

\section{Limitations of the study}

The data quality of our study should be discussed. First of all, it should be mentioned that data were not available from every patient every year. This is why the significance of the data should be nuanced. As an example, in 2015 for the entire cohort, only 486 out of 587 entries (82.79\%) were found for the BMI, only 440 out of 587 (74.96\%) entries were found for FEV1 and only 492 out of 587 (83.82\%) entries were found for intravenous antibiotic therapy. This shows that a significant amount of data is missing and that the quality of the data is negatively affected.

Moreover, the data was collected on one hand by doctors and clinic employees, which makes human bias in the data collection possible. On the other hand, this clinically collected data is entered manually into the computer system, which makes further errors possible and can explain missing data.

Deviations due to anomalies were also found in the patient's follow-up data. These have also affected the quality of the data and thus reduced the representativeness of the data.

\section{Possible explanation for the observed differences}

In our study we could observe the Russian cohort is significantly younger than the German cohort. Average age was 33.57 for Frankfurt, 25.59 for Moscow and for the total cohort 28.14. According to ECFSPR in 2017 mean average age was 22.4 [28] years in Germany and 12.4 [28] years in Russian Federation what confirms our results.

In Moscow 217 (50.70\%) male patients and 211 $(49.30 \%)$ female patients were counted, while in Frankfurt 92 (57.86\%) male patients and 67 (42.14\%) female patients were registered. According to ECFSPR in 2017 in Germany around 52\% were male patients and $48 \%$ were female. In Russian Federation the percentage was similar with about $51 \%$ male patients and $49 \%$ female patients [28]. These results were comparable with our study for Moscow. In Frankfurt the relative amount of male patients was higher than the German average. The observed gender gap could have influenced our results.

According to the number of death patient totalized in our study, there were less deaths in Frankfurt than in Moscow. This can be partially explained by new therapies [29], a better organization [30] and a medicine that becomes more and more detailed and precise due to the economic possibilities and the research. This means that German patients are in a transition phase, where life expectancy increases. Patients in Russia yet are not in this phase. This may be linked to a possible delay in the use of more modern equipment and therapies, as well as probably lower or unevenly distributed financial means.

Moreover the economic structures are different between both countries as well as the regional structures of Frankfurt and Moscow. Russia evaluated recently with the independence from the Soviet Union in 1991 and inherited an extensive centralized system. In 1993 a mandatory health insurance ( $\mathrm{MHI}$ ) was introduced to open up an earmarked stream of funding for health care, but faced lots of fiscal constraints [31]. In Germany, the health system is build up in a different way. The state is organized federally and multiple adapted health care centers were created. This was also reflected in CF management. Since 1995, the German Cystic Fibrosis Quality Assessment project has collected demographic data and outcome parameters, what aims to develop tools for quality management and improve health care [32]. This could also partly explain our results, but has to be confirmed in further studies, where economical, management and organization can be monitored.

\section{Important and new aspect of our study}

Our study is the first one comparing CF patients between both centers of Frankfurt and Moscow. It has confirmed expected differences between surrogate parameters of prognosis in CF patients of both centers. It opens new ways to research possible causes of these gaps. Our results permit to evaluate differences in therapy schemes and the use of various CF medications, in particular CFTR modulator therapies in further studies. Moreover our results show that the necessity of intravenous antibiotic treatment has also to be reexamined. The observed better results in Frankfurt for intravenous therapies in CF patients have to be proved statistically. The relation between the necessity of intravenous antibiotic therapy and a worse outcome for CF-patients has to be discussed. A study published in 2015 questioned the link between both and put other antibiotic treatments (oral therapy or inhaled therapy) on the same acting level [33].

Furthermore, we showed the reasons of these gaps in surrogate markers for CF prognosis have to be investigated. One possible cause could be a possible 
difference in delta F508 mutation or other CF-specific gene mutation distribution [1]. Moreover epidemiologic reasons should be regarded in a larger scale and also compared and evaluated in another study. The socioeconomic differences between both countries should also be taken in consideration.

Our study indicates in particular that different types of drugs, modes of application, frequency of application, treatment regimens and the availability of medication could play a role in CF prognosis.

\section{Conclusion}

We have identified that Frankfurt CF patient's values for surrogate parameters of CF outcome were better than those in Moscow patients in a short time. Further studies should verify this difference on a longer lapse of time including larger data spectrum. First, this will allow establishing a hypothesis explaining this difference. Secondly, this could help to refine therapeutic approaches and to definite new recommendations.

\section{Conflict of Interest Statement}

The authors have declared that no conflict of interest exists.

\section{Declarations of Interest}

None.

\section{Take Home Message}

This study showed a significant difference in prognostic parameters between Frankfurt and Moscow in the cross-sectional analysis for the year 2015.

\section{References}

1. Meng X, Clews J, Ciuta AD, Martin ER, Ford RC (2019) CFTR structure, stability, function and regulation. Biol Chem 400: 1359-1370.

2. Kerem E, Conway S, Elborn S, Heijerman H (2005) Standards of care for patients with cystic fibrosis: A European consensus. J Cyst Fibros 4: 7-26.

3. Gee L, Abbott J, Conway SP, Etherington C, Webb AK (2003) Quality of life in cystic fibrosis: The impact of gender, general health perceptions and disease severity. J Cyst Fibros 2: 206-213.

4. Stephenson AL, Mannik LA, Walsh S, Brotherwood M, Robert R, et al. (2013) Longitudinal trends in nutritional status and the relation between lung function and BMI in cystic fibrosis: A population-based cohort study. Am J Clin Nutr 97: 872-877.

5. Szczesniak R, Heltshe SL, Stanojevic S, Mayer-Hamblett N (2017) Use of FEV1 in cystic fibrosis epidemiologic studies and clinical trials: A statistical perspective for the clinical researcher. J Cyst Fibros 16: 318-326.

6. Bhatt JM (2013) Treatment of pulmonary exacerbations in cystic fibrosis. Eur Respir Rev 22: 205-216.

7. Hayllar KM, Williams SG, Wise AE, Pouria S, Lombard $M$, et al. (1997) A prognostic model for the prediction of survival in cystic fibrosis. Thorax 52: 313-317.
8. Liou TG, Adler FR, Fitzsimmons SC, Cahill BC, Hibbs JR, et al. (2001) Predictive 5-year survivorship model of cystic fibrosis. Am J Epidemiol 153: 345-352.

9. Kerem E, Reisman J, Corey M, Canny GJ, Levison H (1992) Prediction of mortality in patients with cystic fibrosis. N Engl J Med 326: 1187-1191.

10. Keating C, Poor AD, Liu X, Chiuzan C, Backenroth D, et al. (2017) Reduced survival in adult cystic fibrosis despite attenuated lung function decline. J Cyst Fibros 16: 78-84.

11. Nährlich L, Burkhart M, Wiese B (2015) German CFRegistry Annual Report.

12. Ackermann H (2006) BiAS: Biometrische Analyse von Stichproben. ( $8^{\text {th }}$ edn), Frankfurt am M.

13. Yi SW, Ohrr H, Shin SA, Yi JJ (2015) Sex-age-specific association of body mass index with all-cause mortality among 12.8 million Korean adults: A prospective cohort study. Int J Epidemiol 44: 1696-1705.

14. Hayes A, Gearon E, Backholer K, Bauman A, Peeters A (2015) Age-specific changes in BMI and BMI distribution among Australian adults using cross-sectional surveys from 1980 to 2008. Int J Obes (Lond) 39: 1209-1216.

15. (2019) Lungenfunktionstest - Normwerte, Leichter Atmen Gesundheitsportal.

16. Quanjer PH, Stanojevic S, Cole TJ, Baur X, Hall GL, et al. (2012) Multi-ethnic reference values for spirometry for the 3-95-yr age range: The global lung function 2012 equations. Eur Respir J 40: 1324-1343.

17. Ho DE, Imai K, King G, Stuart EA (2011) Matchlt nonparametric preprocessing for parametric causal inference. J Stat Soft 42.

18. Statistisches Bundesamt der Bundesrepublik Deutschland. Körpermaße nach altersgruppen und geschlecht.

19. Shalnova S, Vilkov V, Balanova Y, Muromtseva G, Kapustina A, et al. (2018) P4451comparison of the body mass index in the populations of the Russian Federation and the United States of America during thirty years period. European Heart Journal 39.

20. (2013) Regional Office for Europe. Russian Federation WHO Country Profile, 2013.

21. Rtveladze K, Marsh T, Webber L, Kilpi F, Goryakin Y, et al. (2012) Obesity trends in Russia. The impact on health and healthcare costs. Health 4: 1471-1484.

22. Sachs L (2004) Angewandte Statistik. Berlin, Heidelberg: Springer Berlin Heidelberg.

23. Toutenburg H (1975) Hollander M, DA. Wolfe: Nonparametric statistical methods. John Wiley \& Sons, New York-SydneyTokyo-Mexico City, Biom J 17: 526.

24. Cohen J (2013) Statistical power analysis for the behavioral sciences. ( $\left.2^{\text {nd }} \mathrm{edn}\right)$, Hoboken: Taylor and Francis.

25. Zimmmermann $\mathrm{H}$ (1985) Exact calculation of permutational distributions for two independent samples. Biom J 27: 431434.

26. Corey M, McLaughlin FJ, Williams M, Levison H (1988) A comparison of survival, growth, and pulmonary function in patients with cystic fibrosis in Boston and Toronto. J Clin Epidemiol 41: 583-591.

27. Sharma R, Florea VG, Bolger AP, Doehner W, Florea ND, et al. (2001) Wasting as an independent predictor of mortality in patients with cystic fibrosis. Thorax 56: 746-750. 
28. (2017) European Cystic Fibrosis Society. ECFS Patient Registry - Annual Data Report.

29. Lopes-Pacheco M (2019) CFTR modulators: The changing face of cystic fibrosis in the era of precision medicine. Front Pharmacol 10: 1662.

30. Smaczny C, Eickmeier O, Wagner TOF (2013) Transition in der Pneumologie. Pneumologe 10: 13-19.
31. Popovich L, Potapchik E, Shishkin S, Richardson E, Vacroux A, et al. (2011) Russian Federation. Health system review. Health Syst Transit 13: 1-190.

32. Stern M, Wiedemann B, Wenzlaff P (2008) From registry to quality management: The German cystic fibrosis quality assessment project 1995 2006. Eur Respir J 31: 29-35.

33. Hurley MN, Prayle AP, Flume P (2015) Intravenous antibiotics for pulmonary exacerbations in people with cystic fibrosis. Cochrane Database Syst Rev. 